\title{
Obstructive sleep apnea and tonsillectomy: do we have a new indication for extended postoperative observation?
}

Charles J. Coté MD DABA FAAP, Stephen H. Sheldon DO FAAP

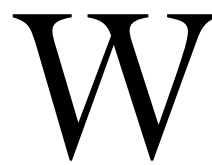

E know that certain subpopulations of children undergoing tonsillectomy or adenotonsillectomy, particularly those with obstructive sleep apnea (OSA), are at greater risk for severe and occasionally life-threatening postoperative complications. Increased oxygen requirements, and a higher incidence of airway obstruction and apnea are often observed in these patients. In this issue of the Canadian Journal of Anesthesia, Koomson et al. have presented us with a possible new concern: children operated upon for tonsillectomy after l p.m. seem to have an increased incidence of desaturation events. ${ }^{1}$ In the USA many insurance companies will not pay for a tonsillectomy unless it is indicated for documented recurrent tonsillitis or OSA. Some insurers will accept a clinical history whereas others require a full sleep laboratory evaluation. A diagnosis established with the latter technology makes the decision for postoperative monitoring easy but a diagnosis made by history alone leaves the anesthesiologist without clear guidance. Most anesthesiologists are unfamiliar with the diagnostic capacity of a complete sleep laboratory (polysomnography) examination.

The accurate diagnosis of respiratory pauses during sleep in children is based on comprehensive attended continuous monitoring of respiratory, cardiovascular, and electroencephalographic (EEG) variables throughout the child's habitual sleep time. The severity of breathing disorders during sleep often varies across the 24-hr day with the highest incidence of pathological apnea occurring during the early morning hours. ${ }^{2}$ Monitoring of sleep is most reliable when performed at night, during the child's major sleep period. "Nap" studies have been shown to lack the sensitivity and specificity to provide accurate informa- tion regarding sleep disordered breathing and OSA syndrome. ${ }^{3}$

Multiple variables are monitored continuously during polysomnography examinations of children. Nasal and oral airflow are evaluated using pressure transduction and capnography. Pressure transduction provides the most accurate measurement of airflow from the nose and mouth. Capnography assesses airflow by continuous measurement of exhaled carbon dioxide. Thermistry (using either a thermistor or thermocouple placed in the air stream at the nose and mouth) demonstrates qualitative airflow by measuring temperature changes that occur between inhalation and exhalation. This method is considered quite accurate in the adult patient however pressure transduction and/or capnography are more reliable in the infant, toddler and child. Respiratory effort during polysomnography is monitored either by piezoelectric crystal strain gauges surrounding the chest and the abdomen or by inductive plethysmography. Strain gauges provide for continuous observation of changes in circumference of the chest and abdomen during the respiratory cycle, while plethysmography assesses changes in volume. Continuous monitoring of respiratory effort by chest wall impedance is rather inaccurate for comprehensive polysomnographic purposes and should be avoided. Oxygen saturation is continuously monitored by pulse oximetry. Gas exchange can also be monitored transcutaneously. Uninterrupted electrocardiogram (EKG) recordings provide feedback of the cardiovascular effects of respiratory pauses. Other variables continuously monitored include $\mathrm{EEG},{ }^{4}$ chin muscle electromyogram (EMG), electro-oculogram, and bilateral anterior tibialis EMG. Esophageal $\mathrm{pH}$ may also be monitored when indicated to assess for possible gastroesophageal reflux. Recording multiple phys-

From the Department of Anesthesiology and Pediatrics, Children's Memorial Hospital, The Feinberg School of Medicine at Northwestern University, Chicago, Illinois, USA.

Address correspondence to: Dr. Charles J. Coté, Department of Pediatric Anesthesiology (Jones 225), Children's Memorial Hospital, The Feinberg School of Medicine at Northwestern University, 2300 Children's Plaza, Chicago, Illinois 60614, USA. Phone: 773-8804414; E-mail: ccote@northwestern.edu 
iological variables accurately identifies pathologic sleep-state-related respiratory changes as well as apneas associated with other significant sleep-related disorders (e.g., sleep-related seizure activity, gastroesophageal reflux).

Isolated pneumography (respiratory effort monitoring by thoracic impedance and heart rate monitoring), as it is often used for postoperative monitoring, should be avoided as a preoperative diagnostic method. ${ }^{5}$ Pneumograms can significantly underestimate the integrity of respiration during sleep. Sensitivity and specificity of pneumography is poor. False-positive and false-negative results are common. Partial airway obstruction may cause false breath detection, and apneas immediately following a sigh or biphasic respiratory efforts are often missed. ${ }^{6}$ In addition, unless nasal and oral airflow are recorded, only central apneas can be detected (unless there are significant cardiovascular effects of the respiratory pause). Central apneas are only some of the breathing-related abnormalities that occur during the sleep period. Thoracic impedance measurements may fail to detect obstructive apneas and can confuse cardio-ballistic artifact (cardiac oscillations) with respiratory effort. In summary, attended polysomnography in the laboratory is the most reliable and effective method for the identification of normal $v s$ abnormal upper airway function during sleep.

Controversy exists regarding interpretation and degree of sleep disordered breathing that would necessitate surgical intervention. Adult normal values are clearer than pediatric ones. It is generally considered that two occluded respiratory efforts in small children are adequate to define an obstructive apnea. ${ }^{7}$ The apnea index (AI) and the respiratory disturbance index are also measurements of presence and severity of OSA syndrome. These indices reflect the frequency of occlusive and partially occlusive respiratory events per hour of sleep. OSA syndrome is considered to be present if the $\mathrm{AI}$ is $\geq 1$, i.e., one or more apnea events per hour of sleep, and can be classified as mild if the $\mathrm{AI}$ is between $\mathrm{l}$ and 5 , moderate if the $\mathrm{AI}$ is between 5 and 10 , and severe if the AI is greater than 10 events per hour of sleep. Nonetheless, clinical assessment of the polysomnogram, degree of sleep disruption, presence of gas exchange abnormalities, presence of EKG changes (dysrhythmias associated with the occlusive respiratory events), and/or co-morbidity (e.g., choanal stenosis) all contribute to determination of the severity of occlusive sleep-disordered breathing and the need for surgical intervention.

The dilemma we face is how do we predict which tonsillectomy patients are at risk for life-threatening complications such as continued apnea and/or hypoxemia postoperatively? In the USA there are five commonly used indications for overnight or extended observation following tonsillectomy: 1) age less than three years; 2) severe OSA documented in a sleep laboratory; 3 ) extreme obesity ( $>95^{\text {th }}$ percentile); 4 ) living a long distance from an adequate health care facility should bleeding or other problem arise; and 5) coming from a disadvantaged home (inadequate care for a postoperative patient, e.g., substance abusing parents, teenage mother, etc.). ${ }^{8}$

Koomson et al. have provided an interesting twist on the postoperative management of children with OSA, i.e., that there is a higher incidence of desaturation events in children operated upon in the afternoon compared with those operated upon in the morning. ${ }^{1}$ With the increasing need to reduce healthcare costs and the restrictions placed on physicians regarding healthcare decisions, particularly in the United States with managed care, there is great pressure to perform surgery as an outpatient. ${ }^{9,10}$ Thus there is a need for physicians and healthcare facilities to balance best practice regarding safety with the need to accomplish this at the least cost.

The tonsillectomy patient presents special risks that relate to surgical issues (bleeding, worsening airway obstruction) and anesthesiology issues (adequate analgesia without adding additional respiratory compromise, prevention of nausea and vomiting). Each of these issues is associated with morbidity and even mortality. All of these concerns together have resulted in a variety of approaches to postoperative management. Some day surgery centres send tonsillectomy patients immediately home; others hold the children for at least four hours (the interval most likely for bleeding to occur). Hospitals tend to admit the children to a $23-\mathrm{hr}$ unit (to avoid the $24 \mathrm{hr}$ "hospital admission") thus observing the child for an extended time (usually not the full $23 \mathrm{hr}$ ) while minimizing the expense to the insurance provider. This latter approach is cost inefficient for the hospital but provides the best safety net for the children.

Analgesia is generally provided with a combination of oral acetaminophen given preoperatively ${ }^{11,12}$ or rectally after induction and low dose morphine (50 $\left.\mu \mathrm{g} \cdot \mathrm{kg}^{-1}\right) .{ }^{13}$ Some anesthesiologists prefer short acting opioids such as fentanyl so as to minimize respiratory depression and then supplement the analgesia with an additional longer acting opioid in the postanesthesia care unit. Ketorolac seemed to be very promising since it provided analgesia without adverse respiratory effects until it was associated with an increased incidence of postoperative bleeding. ${ }^{14}$ The next genera- 
tion of cyclooxygenase- 2 selective inhibitors (COX-2) may be very useful for providing analgesia in this population since they seem to have minimal effects on platelet function and adequately treat moderate levels of pain. ${ }^{15}$ Postoperative nausea and vomiting are generally treated with 5 HT3 antagonists ${ }^{16,17}$ while the postoperative swelling (plus the vomiting) is treated with varying doses of dexamethasone (0.2-0.5 $\mathrm{mg} \cdot \mathrm{kg}^{-1}$ up to $\left.12 \mathrm{mg}\right){ }^{18,19}$ The most common at home analgesic is acetaminophen with codeine.

Unavoidably, the Koomson paper suffers from all the problems associated with a retrospective study, however the observation that there is an increased incidence of desaturation when the tonsillectomy is performed in the afternoon is intriguing and may suggest an additional indication for extended postanesthesia observation including overnight monitoring during the patient's first natural sleep period following anesthesia. The authors also correctly point out that the likelihood for significant desaturation postoperatively was associated with the severity of the OSA syndrome and the degree of desaturation prior to surgery. This finding suggests a strong consideration of polysomnography prior to tonsillectomy in order to identify those children at an increased risk for postoperative complications.

The authors indicate that adverse anesthesia outcomes are associated with weekend surgery and this is thought to be due to decreased staffing. Interestingly the Koomson paper found that there was an increased incidence of desaturation at a time when there is decreased staffing to make such an observation. If anything the likelihood for missing such events is greater with lower staffing levels yet the authors found the reverse! On the other hand, the lower incidence of desaturation following morning surgery may reflect the fact that there are more people around (nurses, parents, doctors, other healthcare providers) any one of which could prevent the patient from falling asleep. There may be other factors that have not been examined such as the fact that senior surgeons tend to get the morning schedule and more junior surgeons get the afternoon time; perhaps surgical technique is another issue. ${ }^{20-23}$ Additional covariates such as body positions during sleep, obesity, gender, and ethnic origin may also be important; these covariates were not all quantitated in the Koomson study. ${ }^{24-27}$ Regardless of the flaws in the Koomson study, they have turned over a rock and now new interesting questions have been raised. We challenge them or other investigators to perform a prospective study that controls for anesthetic technique, surgeon, opioid use, body habitus, ethnic origin, and surgical technique and collects all oximeter and apnea data continuously while also tracking the child's sleep position. It is only with such a carefully controlled prospective large population study that we will be able to sort out if this observation is a new twist or an artifact of inadequate sample size. Our mission as anesthesiologists is to protect the children who are at an increased risk and to gather the information required to convince hospital administrators and insurance companies that some children require more intensive monitoring even though it costs more money to do so.

\section{L'apnée obstructive du sommeil et l'amygdalec- tomie : y a-t-il une nou- velle indication pour une observation postopératoire pro- longée ?}

Nous savons que certaines sous-populations d'enfants qui subissent une amygdalectomie ou une adénoamygdalectomie, surtout celles qui présentent de l'apnée obstructive du sommeil (AOS), sont plus à risque de complications sévères et même graves parfois. Les besoins croissants d'oxygène et une plus forte incidence d'obstruction des voies respiratoires et d'apnée sont souvent observés chez ces patients. Dans le présent numéro du Journal, Koomson et coll. nous présentent un nouveau sujet d'inquiétude possible : les enfants opérés pour amygdalectomie après $13 \mathrm{~h}$ semblent présenter une incidence plus élevée de désaturation. ${ }^{1}$ Aux États-Unis, de nombreuses compagnies d'assurances ne paieront l'amygdalectomie que si elle est indiquée pour des amygdalites répétées ou de l'AOS. Certains assureurs acceptent en preuve une histoire clinique tandis que d'autres exigent une évaluation complète en laboratoire du sommeil. Un diagnostic établi à partir de cette dernière technologie facilite la décision quant au monitorage postopératoire, mais un diagnostic fait à partir de l'histoire seule ne donne pas d'indication claire à l'anesthésiologiste. La capacité diagnostique d'un examen complet du 
sommeil (polysomnographie) est peu connue de la plupart des anesthésiologistes.

Le diagnostic précis de pauses respiratoires pendant le sommeil chez les enfants est fondé sur le monitorage supervisé, complet et continu des variables respiratoires, cardiovasculaires et électroencéphalographiques (EEG) pendant les heures habituelles de sommeil. La sévérité des troubles respiratoires du sommeil varie souvent au cours des $24 \mathrm{~h}$ du jour avec l'incidence la plus importante d'apnée pathologique tôt le matin. ${ }^{2} \mathrm{La}$ surveillance du sommeil est plus fiable lorsqu'elle est réalisée la nuit, pendant la plus importante période de sommeil de l'enfant. Les études portant sur les siestes ont affiché un manque de sensibilité et de spécificité à fournir une information exacte sur les troubles respiratoires du sommeil et sur le syndrome d'AOS. ${ }^{3}$

De nombreuses variables sont enregistrées continûment pendant la polysomnographie chez des enfants. Les débits aériens nasal et buccal sont évalués par transduction de pression et capnographie. La transduction de pression fournit les mesures les plus précises du débit aérien du nez et de la bouche. La capnographie évalue le débit par la mesure continue du gaz carbonique expiré. La thermométrie, réalisée au moyen d'une thermistance ou d'un couple thermoélectrique placé dans le courant d'air nasal et buccal, montre la qualité du débit aérien obtenu par la mesure des changements de température qui surviennent entre l'inspiration et l'expiration. Méthodes assez exactes chez l'adulte, la transduction de pression et / ou la capnographie sont plus fiables chez le nouveauné, le bébé et l'enfant. L'effort respiratoire pendant la polysomnographie est surveillé soit par un tensiomètre à cristal piézo-électrique placé autour du thorax et de l'abdomen, soit par pléthysmographie inductive. Les indicateurs de tension permettent une observation continue des modifications de l'expansion circonférentielle du thorax et de l'abdomen pendant le cycle respiratoire, tandis que la pléthysmographie évalue les changements de volume. Le monitorage continu de l'effort respiratoire par l'impédance de la paroi thoracique est plutôt imprécis pour répondre à des objectifs polysomnographiques complets et ne devrait pas être utilisé. La saturation en oxygène est continuellement surveillée par l'oxymétrie de pouls. L'échange gazeux peut aussi faire l'objet d'un enregistrement transcutané. L'électrocardiogramme (ECG) continu donne un portrait des effets cardiovasculaires des pauses respiratoires. D'autres variables surveillées en continu concernent l'EEG, ${ }^{4}$ l'électromyogramme (EMG) de la houppe du menton, l'électro-oculogramme et l'EMG des muscles jambiers antérieurs bilatéraux. Le pH œsophagien peut être aussi enreg- istré, selon les indications, pour évaluer un reflux gastro-œsophagien possible. L'enregistrement de multiples variables physiologiques permet de déterminer précisément des modifications respiratoires reliées à un sommeil pathologique aussi bien que les apnées associées à d'autres troubles du sommeil significatifs (par ex., l'activité épileptique du sommeil, le reflux gastroœsophagien).

Le tracé des mouvements respiratoires utilisé seul (monitorage de l'effort respiratoire par impédance thoracique et surveillance de la fréquence cardiaque), comme c'est souvent le cas en monitorage postopératoire, devrait être évité comme méthode diagnostique préopératoire. ${ }^{5}$ Les pneumogrammes peuvent sousestimer de façon significative l'intégrité de la respiration pendant le sommeil. La sensibilité et la spécificité de ces tracés sont pauvres. Des résultats faux positifs et faux négatifs sont fréquents. L'obstruction partielle des voies respiratoires peut causer une détection fausse de la respiration et les apnées qui suivent immédiatement un soupir ou des efforts respiratoires biphasiques sont souvent manqués. ${ }^{6}$ De plus, à moins que le débit aérien nasal et buccal ne soit enregistré, seules les apnées d'origine nerveuse centrale peuvent être détectées (sauf s'il y a des effets cardiovasculaires significatifs de la pause respiratoire). Les apnées centrales ne sont qu'une des anomalies reliées à la respiration à survenir pendant le sommeil. Les mesures de l'impédance thoracique peuvent faillir à détecter les apnées obstructives et peuvent confondre l'artéfact cardioballistique (oscillations cardiaques) et l'effort de la respiration. En résumé, la polysomnographie complète est la méthode la plus fiable et la plus efficace d'identification de la fonction normale $v s$ anormale des voies respiratoires pendant le sommeil.

Il existe une controverse quant à l'interprétation de la respiration du sommeil troublé et à la sévérité qui conduirait à une intervention chirurgicale. Les valeurs normales chez un adulte sont plus claires que chez l'enfant. On considère généralement que deux efforts respiratoires occlus chez de jeunes enfants sont suffisants pour définir une apnée obstructive. ${ }^{7}$ L'indice d'apnée (IA) et l'indice de trouble respiratoire sont aussi des mesures de la présence et de la sévérité du syndrome d'AOS. Ces indices traduisent la fréquence d'événements respiratoires occlusifs et partiellement occlusifs par heure de sommeil. Le syndrome d'AOS est considéré comme présent si l'IA est $\geq 1$, c'est-àdire une apnée ou plus par heure de sommeil, et peut être classifié comme léger si l'IA est entre 1 et 5 , modéré entre 5 et 10 et sévère s'il y a plus de 10 incidents par heure de sommeil. Néanmoins, l'évaluation clinique du polysomnogramme, le degré de perturbation 
du sommeil, la présence d'échanges gazeux anormaux, de changements à l'ECG (dysrythmies associées aux événements respiratoires d'origine occlusive) et / ou une co-morbidité (par ex., une sténose choanale) contribuent tous à la détermination de la sévérité du trouble de la respiration d'origine occlusive du sommeil et du besoin d'intervention chirurgicale.

Le dilemme auquel nous faisons face consiste à prédire quels seront, après une amygdalectomie, les patients à risque de complications graves continues comme l'apnée et / ou l'hypoxémie postopératoire ? Aux États-Unis, on utilise généralement cinq indications pour l'observation d'une seule nuit ou l'observation prolongée après une amygdalectomie : 1 ) avoir moins de trois ans ; 2) présenter une AOS sévère révélée pendant une évaluation en laboratoire de sommeil ; 3) souffrir d'une obésité morbide ( $>95^{\mathrm{e}}$ percentile); 4) habiter loin d'un établissement de santé adéquat, si des saignements ou d'autres problèmes survenaient ; 5) provenir d'un milieu défavorisé (soins postopératoires inadéquats, par ex., de parents toxicomanes, d'une mère adolescente, etc.). ${ }^{8}$

Koomson et coll. présentent un retournement intéressant du traitement postopératoire d'enfants atteints d'AOS en affirmant qu'il y a une incidence plus élevée de désaturation chez les enfants opérés pendant l'après-midi comparés à ceux qui sont opérés le matin. ${ }^{1}$ Avec le besoin croissant de réduire les coûts des soins de santé et les restrictions demandées aux médecins face aux décisions concernant les soins à donner, surtout aux États-Unis avec les soins gérés, la pression est plus grande de réaliser des opérations ambulatoires. ${ }^{9}, 10$ Il y a donc un besoin pour les médecins et les établissements de santé d'équilibrer la meilleure pratique au plan de la sécurité avec la nécessité de le faire au meilleur coût.

Le patient d'amygdalectomie présente des risques particuliers qui sont reliés aux conséquences chirurgicales (saignement, aggravation de l'obstruction des voies respiratoires) et aux questions d'anesthésiologie (analgésie suffisante sans ajout de risque respiratoire supplémentaire, prévention des nausées et des vomissements). À chacune de ces préoccupations est associée une morbidité et même une mortalité. Ces préoccupations sont à l'origine de démarches variées de prise en charge postopératoire des patients. Certains centres de chirurgie d'un jour renvoient les patients à la maison immédiatement après l'amygdalectomie ; d'autres gardent les enfants pour au moins quatre heures (surtout pour prévenir les saignements). Des hôpitaux tendent à admettre les enfants dans des unités de court séjour, pour éviter «l'admission hospitalière» de $24 \mathrm{~h}$, ce qui permet alors d'ob- server l'enfant pour une période prolongée, habituellement moins de $23 \mathrm{~h}$, tout en diminuant les coûts pour la compagnie d'assurances. Cette façon de procéder n'est pas rentable pour l'hôpital, mais elle assure une plus grande sécurité aux enfants.

L'analgésie est généralement fournie par une combinaison d'acétaminophène administrée par voie orale avant l'opération ${ }^{11,12}$, ou par voie rectale après l'induction de l'anesthésie, et d'une faible dose de morphine $\left(50 \mu \mathrm{g} \cdot \mathrm{kg}^{-1}\right) .{ }^{13}$ Certains anesthésiologistes préfêrent les opioïdes à action brève comme le fentanyl pour réduire la dépression respiratoire et, ensuite, une analgésie complémentaire avec des opioïdes à action plus longue administrés à la salle de réveil. Le kétorolac semblait très prometteur, puisqu'il produisait une analgésie sans effets secondaires respiratoires jusqu'à ce qu'il soit associé à une incidence accrue de saignements postopératoires. ${ }^{14}$ La nouvelle génération d'inhibiteurs sélectifs de cyclooxygénase-2 (COX-2) pourrait être très utile pour l'analgésie auprès de cette population, car elle semble présenter des effets minimaux sur la fonction plaquettaire et traiter adéquatement les douleurs modérées. ${ }^{15}$ Les nausées et les vomissements postopératoires sont généralement traités avec des antagonistes de $5 \mathrm{HT} 3^{16,17}$ tandis que l'œdème postopératoire, et les vomissements, sont traités avec diverses doses de dexaméthasone $\left(0,2-0,5 \mathrm{mg} \cdot \mathrm{kg}^{-1}\right.$ jusqu'à $\left.12 \mathrm{mg}\right) \cdot{ }^{18,19}$ L'analgésique le plus commun utilisé à la maison est l'acétaminophène avec de la codéine.

L'article de Koomson souffre inévitablement de tous les problèmes associés à une étude rétrospective, quoique l'observation d'une incidence accrue de désaturation dans le cas d'amygdalectomie réalisée dans l'après-midi soit intrigante et constitue une indication supplémentaire pour une surveillance postanesthésique prolongée en court séjour pendant la première période de sommeil naturel après l'anesthésie. Les auteurs ont aussi souligné avec justesse que la possibilité d'une désaturation postopératoire significative était associée à la sévérité du syndrome d'AOS et au degré de désaturation antérieure à l'intervention chirurgicale. Les résultats suggèrent de considérer fortement l'examen polysomnographique antérieur à l'amygdalectomie dans le but de dépister les enfants à risque élevé de complications postopératoires.

Les auteurs indiquent que les conséquences indésirables de l'anesthésie sont associées à la chirurgie du week-end et que cette situation semble liée à une réduction du personnel. Fait intéressant, Koomson a trouvé qu'il y a une plus grande incidence de désaturation au moment où le personnel est moins nom- 
breux pour faire ce genre d'observation. La possibilité de passer à côté de tels incidents est vraisemblablement plus grande avec un personnel réduit, mais les auteurs ont trouvé l'inverse ! Par contre, la plus faible incidence de désaturation après une opération du matin peut illustrer le fait qu'il y ait plus de gens autour (infirmières, parents, médecins et autre personnel de la santé) et que chaque personne puisse empêcher le patient de s'endormir. Il existe peut-être d'autres facteurs, non examinés, comme le fait que les chirurgiens seniors aient tendance à travailler le matin et que les chirurgiens plus jeunes se retrouvent en salle d'opération l'après-midi ; aussi, il faut peut-être considérer la technique chirurgicale comme une autre variable..$^{20-23}$ Des covariables supplémentaires, comme la position du corps pendant le sommeil, l'obésité, le sexe et l'origine ethnique pourraient aussi être importantes, mais elles ne sont pas quantifiées dans l'étude de Koomson. ${ }^{24-27}$ Mis à part les défauts de l'étude, les auteurs ont mis au jour de nouveaux faits et soulevé de nouvelles questions fort intéressantes. Le défi leur est lancé, ainsi qu'à d'autres chercheurs, de réaliser une étude prospective qui tienne compte de la technique anesthésique, du chirurgien, de l'usage d'opiödes, de la morphologie corporelle, de l'origine ethnique et de la technique chirurgicale, et qui recueille toutes les données de l'oxymétrie et de l'apnée de façon continue tout en notant la position de l'enfant pendant le sommeil. Ce n'est qu'avec une étude prospective soigneusement contrôlée, auprès d'une grande population, que nous pourrons établir si cette observation est une nouvelle dimension ou un artéfact lié à un échantillon de la taille insuffisante. Notre mission comme anesthésiologistes est de protéger les enfants qui sont à risque élevé et de recueillir des informations pour convaincre les administrateurs d'hôpitaux et les compagnies d'assurances que certains enfants ont besoin d'une surveillance plus intensive même s'il en coûte plus cher d'agir ainsi.

\section{References}

1 Koomson A, Morin I, Brouillette R, Brown KA. Children with severe OSAS, who have adenotonsillectomy in the morning, are less likely to have postoperative desaturation than those operated on in the afternoon. Can J Anesth 2004; 51: 62-7.

2 Guilleminault C. Obstructive sleep apnea syndrome in children. In: Guilleminault C (Ed.). Sleep and its Disorders in Children. New York: Raven Press; 1987: 213.

3 Anonymous. Clinical practice guideline: diagnosis and management of childhood obstructive sleep apnea syndrome. Pediatrics 2002; 109: 704-12.
4 Rechtschaffen A, Kales A. A Manual of Standardized Terminology, Techniques and Scoring System for Sleep Stages of Human Subjects. Los Angeles: BIS/BRI, UCLA; 1968.

5 National Institutes of Health Consensus Development Conference. Infantile apnea and home monitoring. Bethesda, Maryland, US Department of Health and Human Services: NIH Publication No 87-2950; 1987.

6 Bronillette RT, Morrow AS, Weese-Mayer DE, Hunt CE. Comparison of respiratory inductive plethysmography and thoracic impedance for apnea monitoring. J Pediatr 1987; 111: 377-83.

7 Loughlin GM. Obstructive sleep apnea syndrome in children. Diagnosis and management. In: Loughlin GM, Carroll JL, Marcus CL (Eds.). Sleep and Breathing in Children: a Developmental Approach. New York: Marcel Dekker Inc.; 2000: 625-50.

8 Oddo AR. Healthcare ethics: a patient-centered decision model. J Bus Ethics 2001; 29: 125-34.

9 Modell $\mathrm{JH}$. Health care delivery systems, managed care, and the changing practice of medicine. J Clin Anesth 1998; 10: 347-52.

10 Anderson BJ, Holford NH, Woollard GA, Kanagasundaram S, Mahadevan M. Perioperative pharmacodynamics of acetaminophen analgesia in children. Anesthesiology 1999; 90: 411-21.

11 Anderson B, Kanagasundarum S, Woollard G. Analgesic efficacy of paracetamol in children using tonsillectomy as a pain model. Anaesth Intensive Care 1996; 24: 669-73.

12 Birmingham PK, Tobin MJ, Henthorn TK, et al. Twenty-four-hour pharmacokinetics of rectal acetaminophen in children. An old drug with new recommendations. Anesthesiology 1997; 87: 244-52.

13 Gunter JB, Varughese AM, Harrington JF, et al. Recovery and complications after tonsillectomy in children: a comparison of ketorolac and morphine. Anesth Analg 1995; 81: 1136-41.

14 Sinatra $R$. Role of COX-2 inhibitors in the evolution of acute pain management. J Pain Symptom Manage 2002; 24: S18-S27.

15 Barst SM, Leiderman JU, Markowitz A, Rosen AM, Abramson AL, Bienkowski RS. Ondansetron with propofol reduces the incidence of emesis in children following tonsillectomy. Can J Anesth 1999; 46: 359-62.

16 Fujii $\Upsilon$, Tanaka H, Toyooka $H$. Granisetron reduces vomiting after strabismus surgery and tonsillectomy in children. Can J Anaesth 1996; 43: 35-8.

17 Fujii $\Upsilon$, Tanaka H, Toyooka $H$. Granisetron and dexamethasone provide more improved prevention of postoperative emesis than granisetron alone in children. Can J Anaesth 1996; 43: 1229-32.

18 Splinter $W$, Roberts DJ. Prophylaxis for vomiting by 
children after tonsillectomy: dexamethasone versus perphenazine. Anesth Analg 1997; 85: 534-7.

19 Brown OE, Cunningham MJ. Tonsillectomy and adenoidectomy inpatient guidelines: recommendations of the AAO-HNS Pediatric Otolaryngology Committee. Am Acad Otolaryngol-Head Neck Surg Bulletin 1996; 15: 13-5.

20 Lassaletta L, Martin G, Villafruela MA, Bolanos C, Alvarez-Vicent JJ. Pediatric tonsillectomy: post-operative morbidity comparing microsurgical bipolar dissection versus cold sharp dissection. Int J Pediatr Otorhinolaryngol 1997; 41: 307-17.

21 Isaacson $G$, Szeremeta $W$. Pediatric tonsillectomy with bipolar electrosurgical scissors. Am J Otolaryngol 1998; 19: 291-5.

22 Pizzuto MP, Brodsky L, Duffy L, Gendler J, Nauenberg $E$. A comparison of microbipolar cautery dissection to hot knife and cold knife cautery tonsillectomy. Int J Pediatr Otorhinolaryngol 2000; 52: 239-46.

23 Young C, MacRae J. Tonsillectomy. A comparative study of dissection/snare vs suction-cautery. Can Oper Room Nurs J 2001; 19: 7-11.

24 Stepanski E, Zayyad A, Nigro C, Lopata M, Basner R. Sleep-disordered breathing in a predominantly AfricanAmerican pediatric population. J Sleep Res 1999; 8: 65-70.

25 Fernandes do Prado LB, Li X, Thompson R, Marcus CL. Body position and obstructive sleep apnea in children. Sleep 2002; 25: 66-71.

26 Carroll JL, Marcus CL, Loughlin GM. Disordered control of breathing in infants and children. Pediatr Rev 1993; 14: 51-65.

27 Mazza M, Faia V, Paciello N, Della Marca G, Mazza S. Sleep disorders in childhood: a review. Clin Ter 2002; 153: 189-93. 